\title{
Biology of FGFRL1, the fifth fibroblast growth factor receptor
}

\author{
Beat Trueb
}

Received: 19 September 2010/Revised: 20 October 2010/Accepted: 22 October 2010/Published online: 16 November 2010

(C) Springer Basel AG 2010

\begin{abstract}
FGFRL1 (fibroblast growth factor receptor like 1) is the most recently discovered member of the FGFR family. It contains three extracellular Ig-like domains similar to the classical FGFRs, but it lacks the protein tyrosine kinase domain and instead contains a short intracellular tail with a peculiar histidine-rich motif. The gene for FGFRL1 is found in all metazoans from sea anemone to mammals. FGFRL1 binds to FGF ligands and heparin with high affinity. It exerts a negative effect on cell proliferation, but a positive effect on cell differentiation. Mice with a targeted deletion of the Fgfrl1 gene die perinatally due to alterations in their diaphragm. These mice also show bilateral kidney agenesis, suggesting an essential role for Fgfrl1 in kidney development. A human patient with a frameshift mutation exhibits craniosynostosis, arguing for an additional role of FGFRL1 during bone formation. FGFRL1 contributes to the complexity of the FGF signaling system.
\end{abstract}

Keywords Fibroblast growth factor (FGF) .

Fibroblast growth factor receptor (FGFR) - FGFRL1 .

Heparin · Kidney development · Bone formation

$\begin{array}{ll}\text { Abbreviations } \\ \text { FGF } & \text { Fibroblast growth factor } \\ \text { FGFR } & \text { Fibroblast growth factor receptor } \\ \text { Ig-like } & \text { Immunoglobulin-like } \\ \text { ITIM } & \text { Immunoreceptor tyrosine-based inhibition motif }\end{array}$

B. Trueb $(\bowtie)$

Department of Clinical Research, University of Bern,

Murtenstrasse 35, 3010 Bern, Switzerland

e-mail: beat.trueb@dkf.unibe.ch

\section{Introduction and lines of discovery}

The fibroblast growth factors (FGFs) regulate a diverse variety of cellular functions such as proliferation, differentiation, migration, and apoptosis. FGF signaling is therefore involved in most biological processes, including embryonic development, organogenesis, angiogenesis, wound healing, and tumor formation. Humans and mice possess 18 different FGF ligands (FGF 1-10, FGF 15-23, FGF 19 is the human orthologue of mouse FGF15) and four FGF homologous factors (FGF 11-14) with unrelated functions [1, 2]. The FGFs are monomeric proteins that bind with high affinity to heparan sulfate proteoglycans, whereby their activity is dramatically increased. Together with the heparan sulfate, the FGFs bind to four different FGF receptors (FGFRs) that belong to the receptor tyrosine kinase family [3]. After ligand binding, the FGFRs dimerize and trans-autophosphorylate specific tyrosine residues in the cytoplasmic domain of the receptors. The signal is then transduced to the interior of the cell by various pathways including Ras/MAP kinase, phospholipase $\mathrm{C} \gamma$, PI3-kinase, and STAT. The FGFRs possess three extracellular immunoglobulin(Ig)-like loops, a single transmembrane domain, and an intracellular tyrosine kinase domain [4]. The first Ig-like loop is separated from the second by a stretch of negatively charged residues that are often referred to as the "acidic box." Mutations in FGFRs can cause a number of skeletal disorders such as craniosynostosis syndromes and chondrodysplasias [5]. Somatic mutations in the FGFRs may lead to unrestricted growth and cancer as observed in bladder carcinomas [6] and chronic myeloproliferative diseases [7].

FGFRL1 is the fifth member of the FGFR family [8]. It also contains three extracellular Ig-like domains and a single transmembrane helix (Fig. 1). However, it does not 


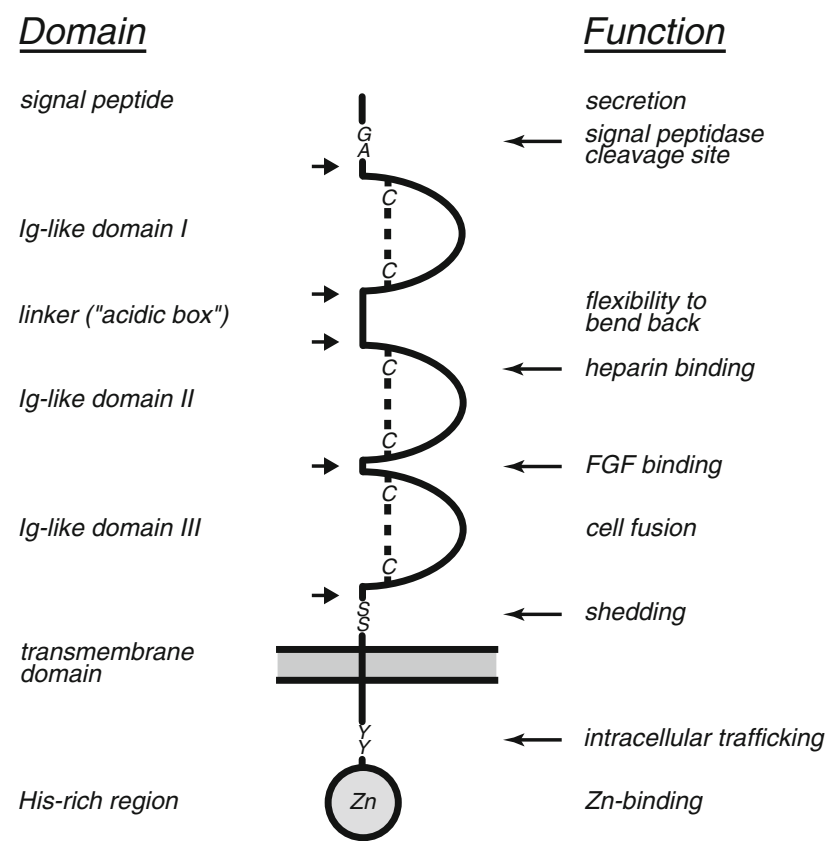

Fig. 1 Schematic drawing of an FGFRL1 monomer. The signal peptide, the three Ig-like domains, the linker ("acidic box"), the transmembrane helix, and the intracellular domain are shown. The disulfide bridges $\mathrm{C}-\mathrm{C}$, the signal peptidase cleavage site $\mathrm{G}-\mathrm{A}$, the site of shedding $\mathrm{S}-\mathrm{S}$, the tandem tyrosine-based motif $\mathrm{Y}-\mathrm{Y}$, and the $\mathrm{Zn}$-binding region are indicated. Short arrows on the left side give the relative positions of introns in the FGFRL1 gene. Note that FGFRL1 is a dimer under physiological conditions

possess an intracellular protein tyrosine kinase domain but instead harbors a C-terminal domain of only 100 residues that cannot signal by transautophosphorylation. The extracellular domain shares $32-35 \%$ sequence identity (39-42\% sequence similarity if conservative replacements are included) with the extracellular domain of the conventional FGFRs. In contrast, the intracellular domain does not show significant similarity with any of the FGFRs or with any other protein.

The novel receptor was described for the first time in the year 2000 and termed FGFRL1 to emphasize its similarity with the members of the FGFR family [8]. At that time, sequences for human FGFRL1 were found in a cartilagespecific cDNA library that had been prepared in order to identify novel regulatory proteins that might play a role in chondrocyte proliferation and differentiation. Shortly thereafter, two other research groups described the same protein and termed it FGFR5 [9, 10]. Kim et al. [9] used the polymerase chain reaction with degenerate primers to amplify cDNAs related to FGFRs. They provided evidence that FGFR5/FGFRL1 was specifically expressed in the human pancreas. Sleeman et al. [10] identified clones for FGFR5/FGFRL1 in a cDNA library prepared from murine peripheral lymph node. When these clones were hybridized to a Northern blot containing RNA from nine different tissues, expression of FGFR5/FGFRL1 was observed not only in lymph nodes but also in kidney, liver, skeletal muscle, heart, and lung. Since no RNA from cartilage and bone had been included, other researchers repeated the Northern blotting experiment with additional tissues [11, 12]. These studies confirmed that FGFRL1 was expressed at relatively high levels in the cartilage of the sternum and the primordia of the vertebrae; at moderate levels in heart, tongue, aorta, lung, kidney, liver, and tendon; and at basal levels in virtually all other tissues. The expression levels increased steadily during mouse embryonic development and became prominent after embryonic day E16 [12, 13]. In general, the expression appeared to be higher in mouse tissues than in chicken or human tissues. By in situ hybridization, the FGFRL1 mRNA was detected in all cartilaginous structures such as the nasal cartilage, the trachea, the ribs, and the primordia of the vertebrae and in some muscles such as the muscles of the tongue and the diaphragm [12]. However, compared to the expression of the classical receptors, expression of FGFRL1 was always relatively weak.

In addition to these studies with human and murine tissues, the FGFRL1 mRNA was also cloned and sequenced from rat [11], chicken [11], frog [14, 15], and fish [16]. Zebrafish (Danio rerio) and pufferfish (Takifugu rubripes) even possess two genes for FGFRL1, fgfrl1a and fgfrl1b, because bony fish have undergone whole genome duplication. The two genes exhibit slightly different expression patterns, a fact that has fostered speculations about subfunctionalization as the driving force to maintain the two genes after duplication [16]. Xenopus laevis, a species that is pseudo-tetraploid, also has two genes for FGFRL1, while Xenopus tropicalis (Silurana) has only one gene since this species is diploid [14, 15]. A protein with $60 \%$ sequence identity to human FGFRL1 was further cloned from the cephalochordate Branchiostoma floridae (lancelet) [15]. Since at that time no similar gene was found in the urochordate Ciona intestinalis, the insect Drosophila melanogaster, or the nematode Caenorhabditis elegans, it was speculated that the FGFRL1 gene might have evolved just before branching of the vertebrate lineage from the other chordates. A few years later, however, the gene for FGFRL1 was also cloned from the echinoderm Strongylocentrotus purpuratus (sea urchin) [17]. Moreover, Bertrand et al. [18] searched for putative orthologues of FGFRL1 in the completely sequenced genomes of several metazoans, and they identified FGFRL1 in the cnidarian Nematostella vectensis (starlet sea anemone) as well as in many other bilaterian species. They concluded that the FGFRL1 gene is present in all metazoans and that it might have evolved together with the other players of the FGF signaling pathway. In flies (e.g., D. melanogaster), nematodes (e.g., C. elegans), and urochordates (e.g., 


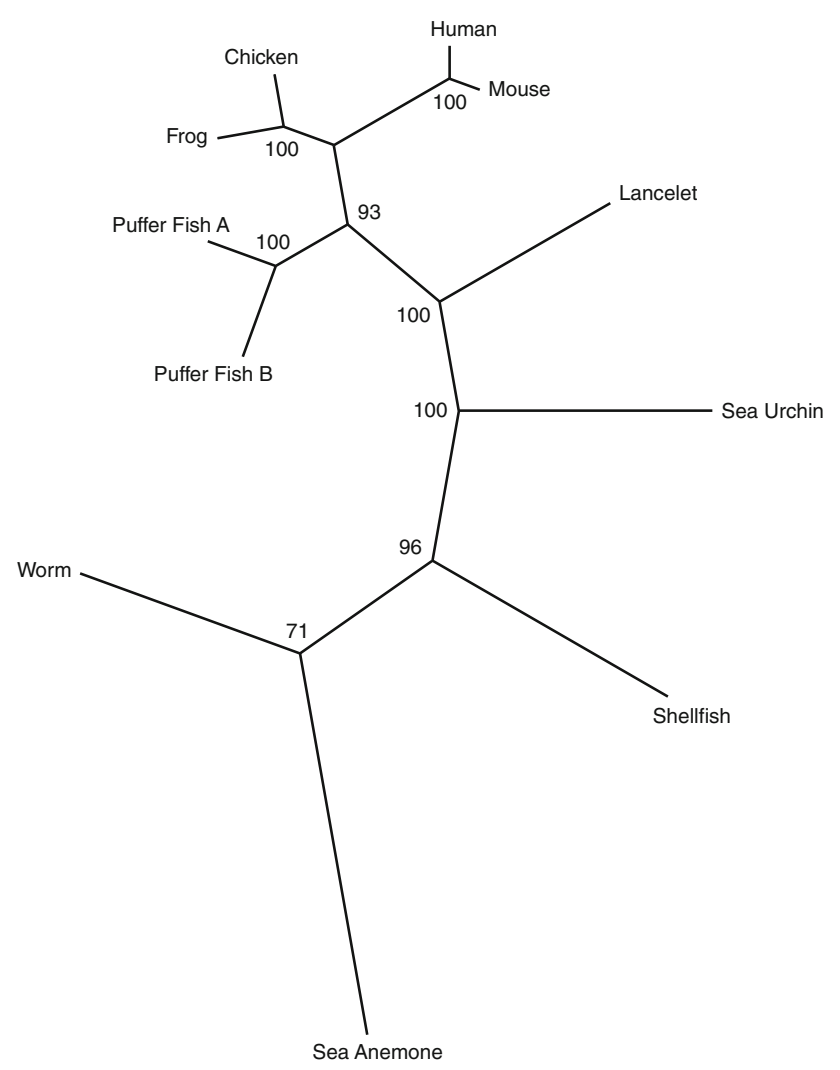

Fig. 2 Phylogenetic tree of FGFRL1 from ten different species. An unrooted tree was built by the neighbor-joining methods. Bootstrap values from 1,000 random replicates are indicated at the nodes (given in \%). The length of the branches inversely correlates with the degree of similarity. Note that only the extracellular domain without signal peptide and transmembrane domain has been used for the phylogenetic analysis

C. intestinalis), the FGFRL1 sequences might simply have escaped our attention because these animals have evolved at a much higher rate. The latter observation is illustrated by the increased level of divergence of protein sequences from flies, nematodes, and sea squirts when compared to the corresponding sequences from cnidaria, annelida, echinoderma, and vertebrates [19]. A phylogenetic tree with the FGFRL1 sequences from ten different species is shown in Fig. 2. This tree is in good agreement with our current understanding of metazoan evolution.

\section{Domain structure}

FGFRL1 is a typical type I transmembrane protein with a single membrane-spanning helix [8-10]. The extracellular domain comprises a signal peptide, three Ig-like domains termed D1, D2, and D3, and a linker region separating domains D1 and D2 (see Fig. 1). Each of the three Ig-domains is stabilized by a single disulfide bond. Following insertion into the endoplasmic reticulum, the signal peptide is cleaved off. The site of cleavage in human FGFRL1 occurs between Gly-17 and Ala-18 as recently demonstrated by mass spectrometric analysis of the isolated extracellular domain from human FGFRL1 [20]. The signal peptide is therefore seven residues shorter than predicted in the Swiss-Prot/UniProt database. At the beginning of domain D2 there is a very basic region that is probably responsible for the interaction of FGFRL1 with heparin [11]. Ten positively charged residues are found at this region of the human protein before the next negatively charged residue follows. The FGF ligands are thought to bind into the groove formed between D2 and D3, as is the case with the classical FGFRs [1], but so far there is no experimental evidence for this assumption. Also in analogy with the conventional FGFRs one may predict that the first Ig domain might have a regulatory or modulating function in that it can fold back onto domain D2 and thereby occupy or sterically hinder the interaction with heparin and/or FGF ligands. The linker between domain D1 and D2 might provide the necessary flexibility for bending. This linker is the least conserved part of the entire extracellular domain in a multiple sequence alignment of FGFRL1 from ten different species (Fig. 3). Thus, the linker will barely have a sequence specific function, but it might rather separate the two domains and provide some flexibility. The best conserved region in the multiple sequence alignment of Fig. 3 is domain D3. Whether this conservation is a consequence of FGF ligand binding is not known. The human FGFRL1 sequence contains four glycosylation sites for the attachment of asparagine-linked carbohydrates that are conserved in all species except the starlet sea anemone. Three of these sites are occupied by carbohydrates as indirectly demonstrated by mass spectrometric analysis [20]. These glycosylation sites are found at slightly shifted positions also in the sea anemone sequence [18], while the fourth site of the human sequence does not have a counterpart in the sea anemone. The attachment of three to four carbohydrate chains is consistent with the observed difference in molecular mass $(10 \mathrm{kDa})$ when FGFRL1 synthesized by HEK293 cells in the presence of the glycosylation inhibitor tunicamycin is compared to that synthesized in the absence of the inhibitor [21].

The intracellular domain of FGFRL1 is only 100 amino acids in length and does not contain any protein tyrosine kinase domain. In a multiple alignment of ten vertebrate sequences, this is the least conserved domain (Fig. 4). The only convincing conservation is found at the very $\mathrm{C}$-terminal end where a peculiar histidine-rich sequence is present. In the human sequence, ten histidine residues alternate with other residues, often threonine (479-HTHTH THSHTHSHVEGKVHQHIHYQC-504). Recently, it has been noted that this histidine-rich sequence can interact with zinc and nickel ions [17]. By atomic absorption, the 


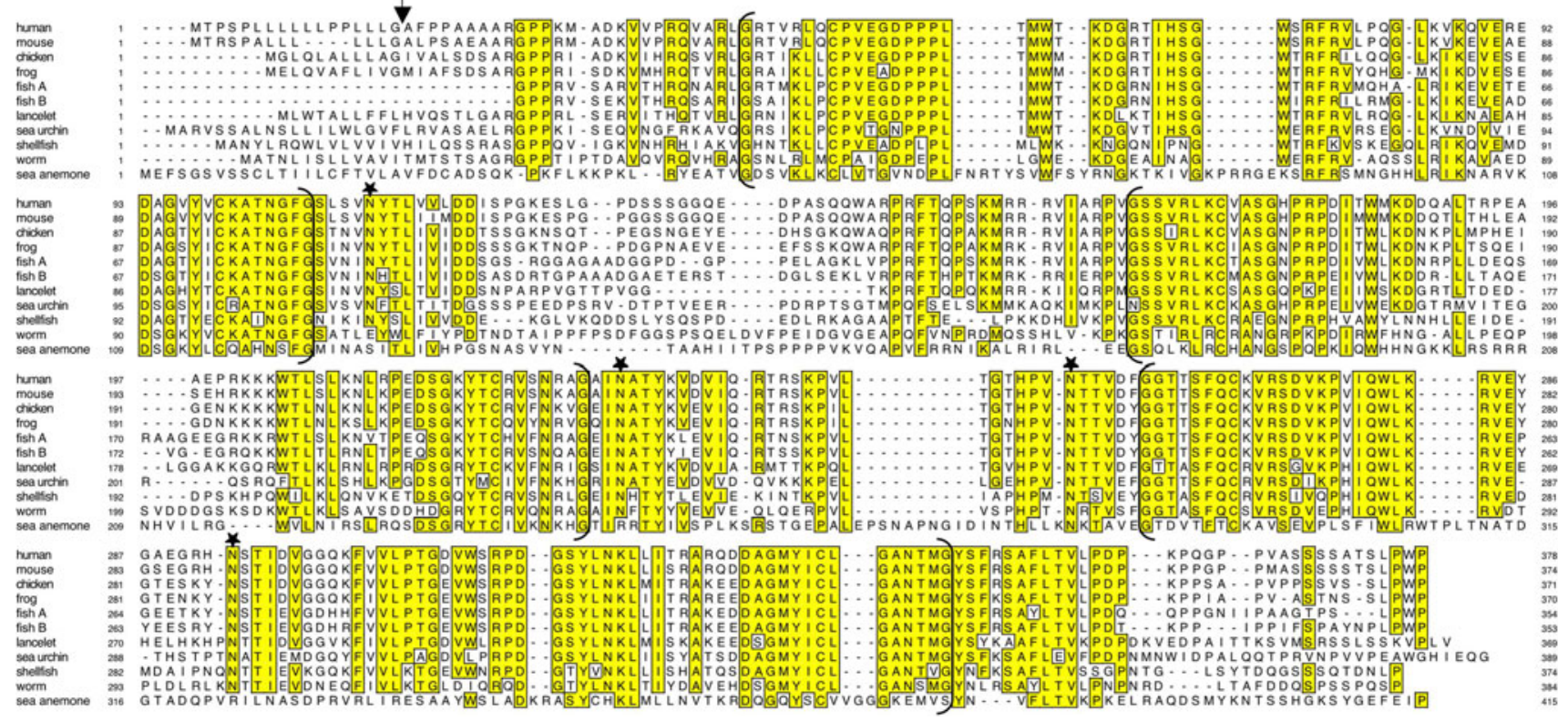

Fig. 3 Alignment of the extracellular domain of FGFRL1 from ten different species. The signal peptidase cleavage site of human FGFRL1 is indicated by an arrow. The three Ig-like domains are shown by brackets. Glycosylation sites NXT in the human sequence are indicated by asterisks. The accession numbers are as follows: human AJ277437, mouse AJ293947, chicken AJ535114, frog
(Silurana tropicalis) AJ616852, fish A (T. rubripes A) BN000669, fish B (T. rubripes B) BN000670, lancelet (B. floridae) AJ888866, sea urchin ( $S$. purpuratus) FN252817, shellfish (Lottia gigantea) 4236761:246, worm (Capitella capitata) 170033, sea anemone (N. vectensis) 204525

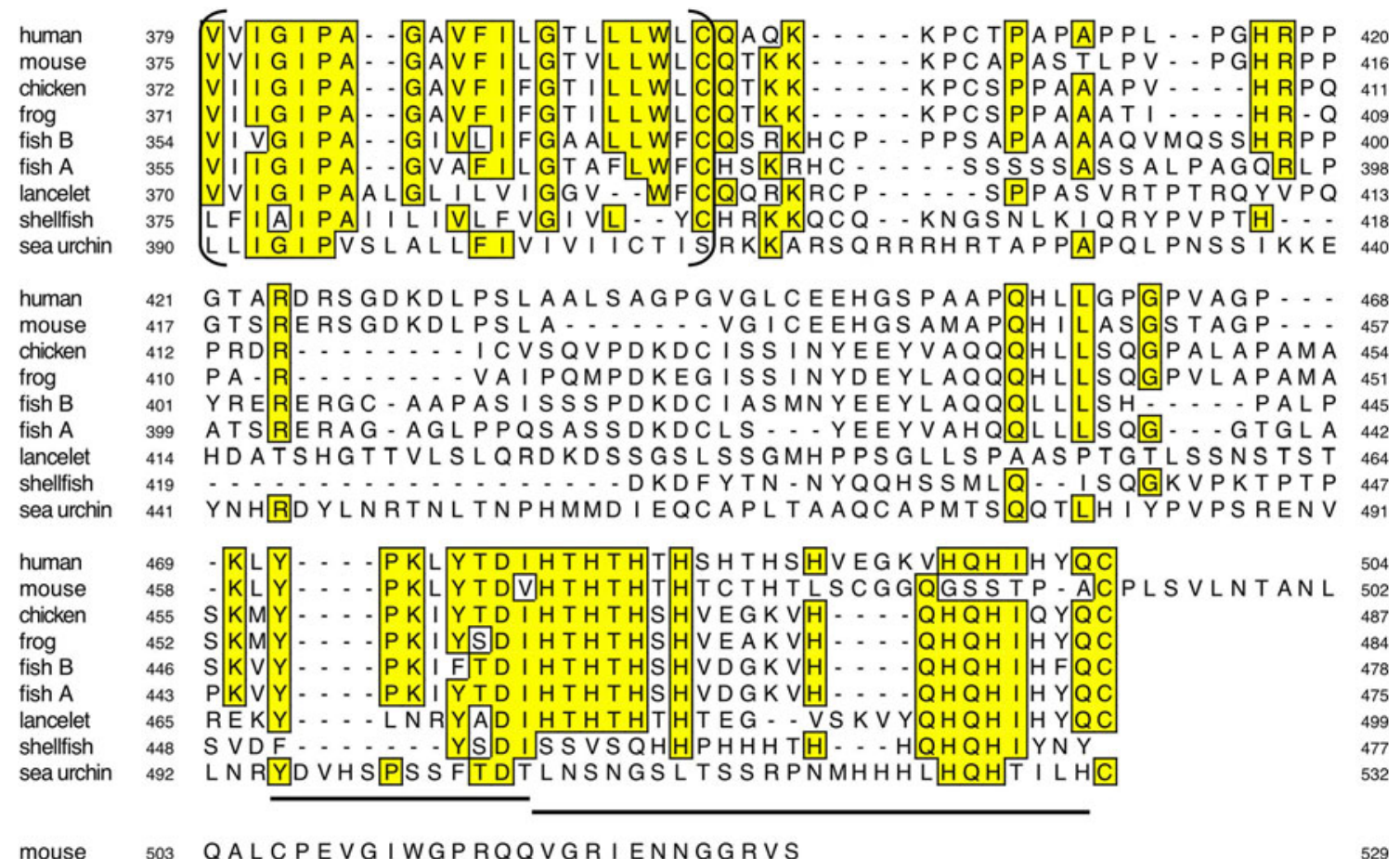

Fig. 4 Alignment of the FGFRL1 sequences for the transmembrane segment and the intracellular domain from eight different species. The transmembrane helix is marked by brackets. The tandem tyrosine-based motif is underlined, as is the histidine-rich sequence. Accession numbers are as given in the legend to Fig. 3. The sequences from worm and sea anemone have not been included since these sequences are much longer and did not align properly. Note that the mouse sequence appears to have suffered a frameshift mutation at residue 475 during evolution $[10,11,13]$ 
human sequence was demonstrated to bind three zinc atoms. The intracellular domain can therefore be purified by chromatography on a nickel affinity column after synthesis in a bacterial expression system, a procedure that is usually employed to purify recombinant proteins with an artificial histidine tag. The histidine-rich sequence is preceded by the tandem tyrosine-based motif PKLYPKLYTDI [10, 22]. This sequence resembles the tyrosine-based motifs YХХФYХХФ (where $\Phi$ is a bulky, hydrophobic amino acid) that are well-known mediators of endocytosis and transmembrane protein trafficking. In fact, truncation experiments and in vitro mutagenesis demonstrated that both the histidine-rich sequence and the tandem tyrosinebased motif act as signals for trafficking of FGFRL1 from the plasma membrane to endosomes and lysosomes [22]. When they were deleted or mutated, FGFRL1 was not efficiently internalized and stayed at the plasma membrane for a prolonged period of time.

A peculiar observation was made with the $C$ terminus of FGFRL1 from rodents. Compared to the human sequence, the mouse and the rat $\mathrm{C}$ terminus must have suffered a mutation during evolution, which led to the replacement of part of the histidine-rich sequence by 54 unrelated residues (see Fig. 4) [10, 11, 13]. Nevertheless, the unrelated sequence must be able to compensate in some way for this mutation since the mouse Fgfrl1 protein shows the normal subcellular distribution. In particular, it does not stay at the plasma membrane for a prolonged period of time as the truncated protein described above [22].

Recently it has also been observed that FGFRL1 can be shed from the plasma membrane [20]. Shedding was first noted in cultures of myoblasts that were allowed to differentiate into myotubes. In such cultures, virtually no FGFRL1 was detected at the cell membrane but relatively large amounts of a soluble form of FGFRL1 accumulated in the cell culture media. The shed protein was also found in the conditioned media of HEK293 cells that overexpressed FGFRL1. The exact cleavage site could be localized by mass spectrometry to the center of a group of four serine residues in the proximity of the transmembrane domain, but so far the identity of the protease responsible for shedding has not yet been elucidated [20]. Experiments with proteinase inhibitors suggested that the enzyme was not related to furin, $\beta$-secretase, or to a matrix metalloproteinase such as ADAM9.

When the overall structure of FGFRL1 was compared to all proteins that are encoded by the human genome, 42 structurally related proteins with a signal peptide, three Ig-like domains, and a single transmembrane helix were identified [23]. These proteins fall into seven different families, namely FGFRs, Fc receptor-like proteins, IL-1 receptor-like proteins, KIRs, nectin-like proteins, sialic acid binding lectins (SIGLECs), and signal regulatory proteins (SIRPs). It remains to be determined whether the structural similarity found between FGFRL1 and all these proteins will also extend to functional similarities.

\section{Gene structure}

The human FGFRL1 gene is found on the short arm of chromosome 4 in band 4p16.3 [8], the mouse Fgfrl1 gene on chromosome 5 in band E3-F [13]. Both genes comprise seven exons. The first exon contains only the $5^{\prime}$ noncoding sequences and was numbered 0 in one publication [24]. The other exons, usually numbered 1-6, code for a separate domain each, exon 1 for the signal peptide, exon 2 for Ig domain D1, exon 3 for the linker (acidic box), exon 4 for Ig domain D2, exon 5 for Ig domain D3 and exon 6 for the transmembrane domain and the intracellular portion. All these exons are flanked by introns of splice phase 1 where the codons for the amino acids are interrupted after the first nucleotide. The exon/intron structure is therefore much simpler than that of the classical FGFRs, which possess up to 19 exons. In these receptors, nine exons are needed to code for the protein tyrosine kinase domain that is missing in FGFRL1. Furthermore, FGFR1, FGFR2, and FGFR3 can be alternatively spliced in the IIIB/IIIC region, a process that requires three separate exons to code for domain D3 [1-3]. Thus, the only additional difference between the exon/intron structure of the FGFRL1 gene and those of the classical receptors concerns domain D2, which is encoded by a single exon in FGFRL1 and by two exons in the classical receptors. However, some deviation from the prototype of the FGFRL1 gene structure is found in several animals including lancelet and pufferfish where the D2 domain is also encoded by two exons [15, 16, 18]. Since the splice phase of the inserted intron is of a different type (splice phase 0, which does not interrupt the codon for the amino acid), it is likely that this intron was inserted into the FGFRL1 gene later during evolution.

Alternative splicing has also been observed with the FGFRL1 gene. Sleeman et al. [10] described the existence of two additional mRNA species that were lacking the sequences for either the first Ig domain or the first Ig domain plus the acidic box. The relevance of this alternative splicing event is not known and no tissue-specific expression has yet been published.

\section{Binding of FGF ligands}

There is ample evidence that FGFRL1 does in fact interact with FGF ligands. The first speculations came from modeling studies in which the polypeptide chain of FGFRL1 was superimposed on the solved three-dimensional 
structure of FGFR1 [10]. This comparison demonstrated that many of the residues that are involved in the interaction with FGF ligands are well conserved in FGFRL1. As previously shown, the FGFRs interact with the ligands primarily via their domains D2 and D3 and the interconnecting linker D2-D3 $[25,26]$. The contacts between the FGFs and the D2 domain as well as the D2-D3 linker are thought to be involved in general binding of the ligands, while the contacts with the D3 domain appear to control the ligand specificity of the receptor. Figure 5 shows an alignment of the extracellular domains from human FGFR1 and FGFR2, whose structures have been solved [25, 26], with that from human FGFRL1. In the FGF2/FGFR2 complex, residues Leu-166, Ala-168, and Pro-170 of D2 make hydrophobic contacts with the ligand. In the aligned FGFRL1 sequence, these residues are conserved as Val-159, Ala-161, and Pro-163. From the D2-D3 linker, Arg-251 forms an important hydrogen bond with the ligand. This residue is conserved as Arg-241 in FGFRL1. In the D3 domain of FGFR2, several hydrogen bonds are formed between residues Asp-283, Gln-285, Val-317, Asn318, and Asp-321 and several sites of the FGF ligand. In FGFRL1, two of these residues are conserved as Asp-273 and Val-311. Since Gln-285, Asn-318, and Asp-321 are not conserved and since these bonds control mainly the ligand specificity, one may speculate that FGFRL1 might have other specificities than FGFR2 and FGFR1.

A direct interaction was experimentally demonstrated with FGF2 and recombinant mouse FGFRL1 (FGFR5) that carried an Fc-tag [10]. When the receptor was incubated with FGF2 and then precipitated with protein G-Sepharose, the precipitates contained nearly stoichiometric amounts of FGF2. This interaction was specific since FGF7 or EGF could not be precipitated in control experiments [10]. In a subsequent publication, radiolabeled FGF2 and recombinant chicken FGFRL1 with a His-tag were utilized for a similar experiment [11]. The radiolabeled growth factor bound to FGFRL1 and was specifically co-precipitated with nickel agarose beads. A dissociation constant $K_{\mathrm{d}}$ of $6 \mathrm{nM}$ was determined for the FGF2/FGFRL1 complex. More recently, a dot blot assay was utilized to test all commercially available FGFs for their ability to bind to recombinant human FGFRL1 that had been produced in HEK293 cells with an myc tag [20]. The growth factors were spotted onto a nitrocellulose membrane and incubated with the soluble FGFRL1. After washing, bound receptor was detected with anti-myc antibodies. Strong binding was observed with FGF3, FGF4, FGF8, FGF10, and FGF22, whereas virtually no binding was obtained with FGF1, FGF6, FGF7, FGF9, FGF12, FGF16, FGF19, FGF20, and FGF21. FGF2, FGF5, FGF17, and FGF23 showed intermediate binding. Some of these results were verified in a cell-based ligand binding assay. FGF1, FGF2, FGF3, and FGF12 were fluorescently labeled with DyLight 547. HEK293 cells that stably expressed FGFRL1 on their cell surface were incubated with the fluorescent ligands. Only FGF2 and FGF3 bound to the cell surface, while FGF1 and FGF12 did not bind [20, 22]. For FGF3, the dissociation constant $K_{\mathrm{d}}$ was directly determined by surface plasmon resonance. The ligand was coupled to the surface of a Biacore sensor chip and increasing concentrations of soluble FGFGRL1 were injected over the chip [22]. Typical

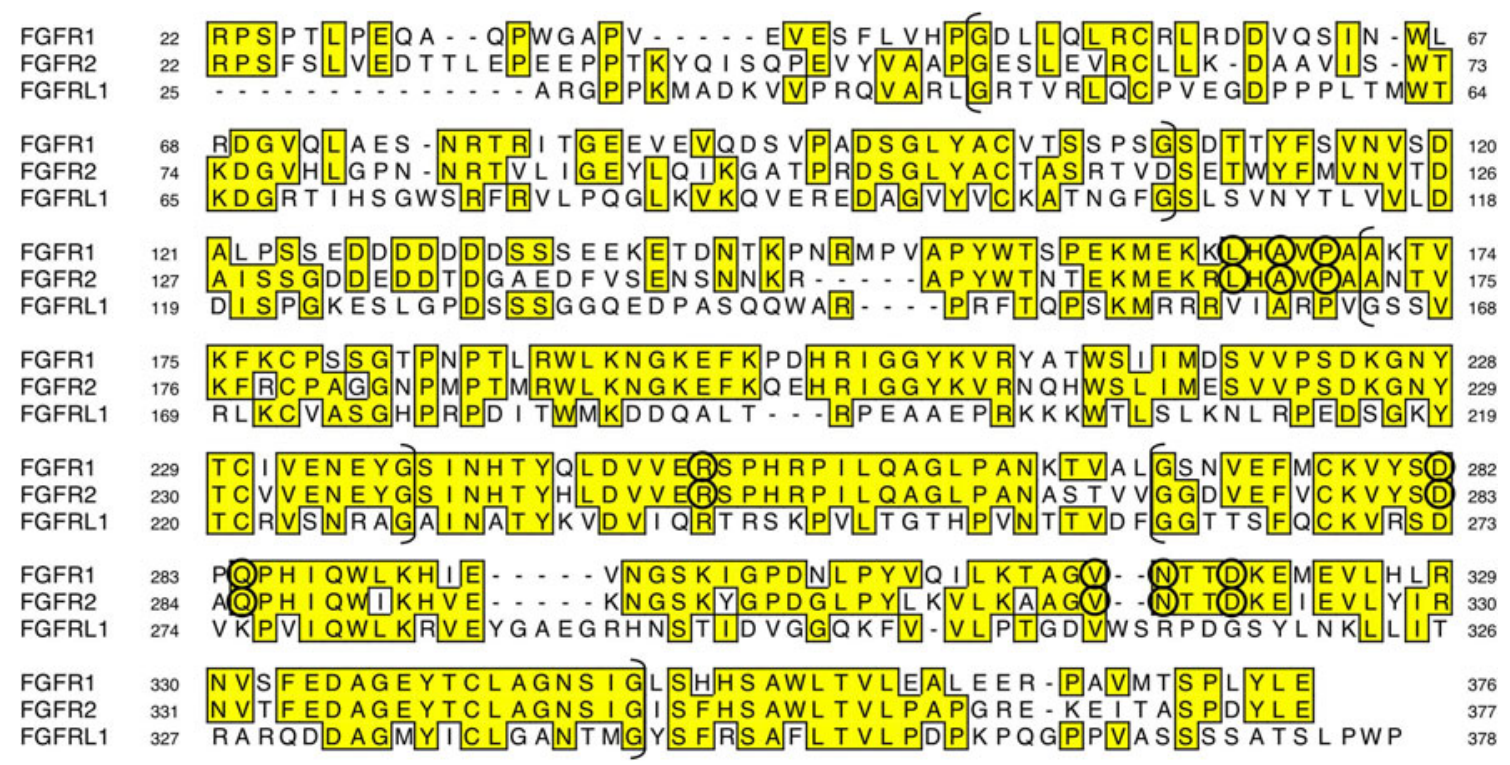

Fig. 5 Alignment of the FGF ligand binding sites from human FGFR1 (NP 075598) and human FGFR2 (NP 000132) with the extracellular domain of human FGFRL1 (AJ277437). Identical residues are boxed. The three Ig-like domains are shown by brackets. Residues that are involved in ligand binding in the FGF1/FGFR1 and FGF2/FGFR2 complex are encircled 
interaction curves with relatively quick association and slow dissociation constants were observed, from which a $K_{\mathrm{d}}$ of $4 \mathrm{nM}$ was determined. This affinity is at least one order of magnitude higher than the affinities published for most FGFs and their cognate receptors. Such $K_{\mathrm{d}}$ values have been described so far only for mutant receptors as they are found in Pfeiffer, Apert, and Muenke craniosynostosis syndromes [27]. Many cases of these syndromes are caused by gain-of-function mutations occurring in the D2-D3 linker, either at Ser-252 (S252W) or Pro-253 (P253R). The mutant receptors exhibit affinities that are roughly one order of magnitude higher than those of the wild-type receptors (e.g., $4 \times 10^{-9}$ for FGF2/FGFR2c $\mathrm{P} 253 \mathrm{R})$ and therefore in the region of the $K_{\mathrm{d}}$ determined for FGFRL1 [27]. In this context, it should also be noted that FGFRL1 has an arginine residue at position 243 that exactly matches the gain-of-function mutation P253R detected in Apert syndrome (Fig. 5). This substitution could therefore be responsible for the unexpectedly high affinity of FGF3 for FGFRL1.

Together, the co-precipitation experiments, the ligand dot blot assays, the cell-based ligand binding studies, and the plasmon resonance measurements clearly demonstrate that FGFRL1 is a genuine FGF receptor with high affinity for some FGF ligands.

\section{Heparin binding}

The classical FGFRs bind to heparin and heparan sulfate, and this heparin binding activity is believed to be important for receptor dimerization and subsequent signaling [1, 3, 28]. Already in the year 1993, the major heparin binding site of FGFR1 was traced to a basic peptide of 18 residues occurring at the beginning of Ig-like domain D2 [29]. Several years later, the dissociation constants $K_{\mathrm{d}}$ were determined for the heparin/FGFR1 $(63 \mathrm{nM})$ and the heparin/FGFR2 complexes (13 nM) [30].

Similar to the classical receptors, FGFRL1 also interacts with heparin. This interaction has enabled the authors to purify recombinant protein from conditioned media of several cell lines by affinity chromatography $[11,21])$. The heparin affinity of FGFRL1 appears to be substantially higher than those of the classical receptors since an ionic strength corresponding to $600-700 \mathrm{mM} \mathrm{NaCl}$ is required to displace FGFRL1 from a heparin column [21] as compared to $330 \mathrm{mM} \mathrm{NaCl}$ (FGFR1 [30]), $430 \mathrm{mM} \mathrm{NaCl}$ (FGFR2 [30]), 300-400 mM NaCl (FGFR4 [31]), and $200 \mathrm{mM}$ $\mathrm{NaCl}$ (FGFR3; Zhuang, personal communication) that are required to displace the other receptors from heparin columns. From the solved three-dimensional structure, it is known that heparin binds into a positively charged canyon formed by domain D2 and FGF [28]. A similar positively charged groove can also be predicted by three-dimensional modeling for the FGFRL1 polypeptide chain. The major cluster of positively charged arginine and lysine residues occurs at the beginning of domain D2. When selected residues from this cluster were exchanged with uncharged or negatively charged residues by in vitro mutagenesis, the mutant protein interacted with heparin-Sepharose with reduced affinity and required only $500 \mathrm{mM}$ instead of $700 \mathrm{mM} \mathrm{NaCl}$ for displacement [22]. Preliminary measurements with an optical biosensor produced a $K_{\mathrm{d}}$ of $9 \mathrm{nM}$ for the heparin/FGFRL1 complex (Zhuang and Trueb, unpublished observation).

\section{Potential ways of signaling}

FGFRL1 cannot signal by transautophosphorylation as there is no intracellular tyrosine kinase domain. FGFRL1 might therefore function as a simple decoy receptor that binds FGF ligands and sequesters them away from the conventional FGFRs [10, 11, 20]. Similar decoy receptors have been described in the literature, in particular in the immune system, where they modulate cellular responses to cytokines. An example would be the IL-1 receptor 2 (IL-1 R2), which binds IL-1 but does not contain the intracellular TIR (TOLL/IL-1 receptor) domain required for signal transduction [32]. Thus, IL-1 R2 sequesters ligands from the signaling receptor IL-1 R1 and reduces IL-1-mediated inflammatory signals.

An alternative way to function would be that FGFRL1 dimerizes with a conventional FGFR and blocks in this way transautophosphorylation since the dimeric complex would have only one tyrosine kinase domain. This possibility seems unlikely because FGFRL1 is known to form constitutive homodimers even in the absence of FGF and heparin [21]. Moreover, FRET analysis did not reveal any evidence for the existence of heterodimers formed between FGFRL1 and FGFR1-4 [21].

Another possibility would be that FGFRL1 accelerates the internalization and degradation of the actively signaling receptors. A prerequisite for this possibility would be that it localizes to the same subcellular compartments as the conventional receptors. When FGFRL1 is internalized and sorted to the endosomes/lysosomes, for instance by its histidine- and tyrosine-based motifs, it could drag along some of the actively signaling receptors. This would be facilitated when a single heparan sulfate chain interacted with both FGFRL1 and the classical FGFRs. At present such a mechanism is purely speculative, and no evidence has been reported in favor of it.

A further possibility would include that FGFRL1 recruits other signaling molecules such as tyrosine phosphatases to the sites where the other signaling receptors are 
located. The peculiar intracellular domain with its tandem tyrosine-based motif and its histidine-rich sequence could be instrumental in the recruitment of such phosphatases. If the phosphatases were brought into close contact to the signaling receptors, they could remove phosphate groups from the latter and attenuate signaling. A similar mechanism has been described in the immune system. Several transmembrane proteins including the Fc receptors [33], the killer cell Ig-like receptors (KIRs) [34], and the SIGLECs [35] harbor ITIM sequences (immunoreceptor tyrosine-based inhibition motifs) in their C-terminal intracellular domains. These ITIMs can become phosphorylated by src family kinases and subsequently bind SH2-containing phosphatases such as SHP-1 and SHP-2 (phosphotyrosine phosphatase 1 and 2) [36, 37]. The phosphatases can then dephosphorylate the cytoplasmic domains of the adjacent receptors and in this way inhibit activation of the cells. However, up to the present moment no phosphorylation of the intracellular domain of FGFRL1 has been observed.

It is obvious that the above list of potential signaling mechanisms is not exhaustive. Moreover, the proposed ways of signaling are not mutually exclusive. For example, it is well conceivable that the extracellular domain of FGFRL1 acts as a decoy receptor while the intracellular domain acts as a docking site for phosphatases.

\section{Biological effects of FGFRL1}

FGFRL1 exerts a variety of seemingly different effects on cells in culture that are difficult to reconcile by a single functional activity. On the one hand, FGFRL1 inhibits cell proliferation and promotes cell differentiation. On the other hand, it induces cell adhesion and appears to be involved in cell-cell fusion.

The first effect was observed with MG63 osteosarcoma cells that had been transfected with expression constructs for FGFRL1 [11]. When compared to control cells treated with anti-sense constructs, cells transfected with sense constructs proliferated at a much slower rate. It was therefore concluded that FGFRL1 exerted a negative effect on cell proliferation. These experiments were later repeated with an inducible system utilizing HEK293-TetOn cells that express FGFRL1 from a construct, which is under the control of the Tet transactivator [38]. In the absence of the inducer doxycycline, these cells proliferated like normal HEK293 cells. In the presence of the inducer, the cells stopped growing and eventually started to die. Thus, FGFRL1 has antiproliferative effects, at least when overexpressed in cell culture.

A negative effect on cell proliferation and a positive effect on cell differentiation are also consistent with the relative expression of FGFRL1 during differentiation of myoblasts in vitro [24]. When $\mathrm{C} 2 \mathrm{C} 12$ cells or primary myoblasts were grown in culture under proliferative conditions (i.e., in the presence of FGF2 or relatively high serum concentrations), the expression of FGFRL1 was minimal. However, when the serum or the growth factors had been removed and the cells were allowed to differentiate into multinucleated, postmitotic myotubes, the expression of FGFRL1 was sharply up-regulated. Thus, FGFRL1 expression correlated directly with the differentiation state of the cells.

In addition to the antiproliferative and proapoptotic effects observed in cell culture, FGFRL1 also exerts a profound effect on cell adhesion. The protein is usually found at sites of cell-cell contact [21]. When coated on plastic surfaces, the extracellular soluble domain of FGFRL1 promoted adhesion of various mammalian cells lines including HEK293, 3T3, and CHO. No similar effect was observed with the extracellular, soluble domain of FGFR1. It is likely that this adhesion-promoting activity of FGFRL1 is mediated by heparan sulfate proteoglycans expressed at the surface of the cultivated cells for it was specifically blocked by the addition of soluble heparin or by mutation of the heparin-binding site in the D2 loop of the immobilized FGFRL1 [21].

Recently, it has also been observed that FGFRL1 has a peculiar effect on cell-cell fusion [38]. The fusion of mammalian cells into large syncytia is a well controlled process that is restricted to a limited set of cells, including gametes, macrophages, myoblasts, and trophoblasts [39, 40]. Steinberg et al. [38] demonstrated that the FGFRL1 receptor, when overexpressed, was capable of fusing $\mathrm{CHO}$ cells into multinucleated syncytia comprising several hundred nuclei. A particularly strong effect was observed with FGFRL1 $\Delta \mathrm{C}$ (a construct that lacked the intracellular domain but still retained the transmembrane domain), but cell fusion could also be induced with the full-length protein. Truncation experiments further demonstrated that Ig domain D3 and the transmembrane domain were both necessary and sufficient for the fusion activity. The novel receptor was even able to fuse heterologous cells, such as HEK293 or HeLa cells, with untransfected CHO cells [38]. FGFRL1 is therefore the first mammalian protein that is capable of inducing syncytium formation of heterologous cells in vitro. It is possible that this activity is involved in the differentiation process of myoblasts into multinucleated myotubes.

\section{Animal models}

The first animal experiments with FGFRL1 were performed by Cebria et al. [41] in the planarian Dugesia 
japonica. These flatworms possess two FGFRs, termed DjFGFR1 and DjFGFR2 [42], and a single FGFRL1 termed nou-darake (Japanese for "brains everywhere"). Similar to vertebrate FGFRL1, the nou-darake protein has three extracellular Ig-like domains (the membrane proximal domain D3 was not indicated in the original publication), a transmembrane domain, and a relatively short intracellular domain [41]. However, the intracellular domain does not contain the histidine-rich sequence typical of vertebrate FGFRL1. Nou-darake is specifically expressed in the head region of the animals. Its function was investigated by RNA interference with dsRNA that was injected directly into the animals. Interestingly, depletion of nou-darake mRNA resulted in the formation of ectopic brain tissue throughout the body, therefore the name "brain everywhere." The ectopic brain formation could be suppressed by the simultaneous injection of dsRNAs against the two DjFGFRs, suggesting that nou-darake regulates signaling by these FGFRs in a negative way. The authors speculated that FGF ligands might act as brain-inducing factors and that the diffusion radius of these factors is restricted by nou-darake to the planarian head [41]. This interpretation is consistent with a decoy function of noudarake.

Functional studies were also performed in zebrafish embryos by Hall et al. [43]. These authors found evidence for an essential role of FGFRL1 during craniofacial skeletogenesis. Depletion of the zebrafish mRNAs for Fgfrlla and Fgfrl1b with morpholino constructs caused a severe reduction in the formation of the cartilage elements in the developing pharyngeal arches, especially in branchial arches 3-5. The authors concluded that Fgfrl1 is required for gill cartilage development. Moreover, they provided evidence that Fgfrl1 might control the expression of $\mathrm{Gcm} 2$ (glial cells missing 2), a transcription factor that had previously been implicated in the formation of gill cartilage $[44,45]$.

Another study was performed in Xenopus embryos [20]. From previous experiments it was known that overexpression of a truncated form of FGFR1, termed XFD, disrupted the normal development of Xenopus embryos by interfering with FGF signaling in a dominant-negative way [46]. The resulting animals showed gastrulation defects that affected trunk and tail and impaired notochord and muscle development. In the more recent experiments, injection of human or mouse FGFRL1 mRNA into Xenopus blastomers led to the same posterior-ventral truncation phenotype of the animals [20]. The dominant-negative effect of the injected FGFRL1 mRNA could be reversed by co-injection of mRNA for FGFR1. Thus, these experiments clearly demonstrate that FGFRL1 interferes with FGF signaling. Similar experiments with Хenopus embryos have also been performed with the nou-darake mRNA [41]. In this context, it is worth emphasizing that the negative effects on FGF signaling were even observed when RNA for the soluble, extracellular form of FGFRL1/nou-darake was injected into the animals. It is therefore likely that FGFRL1 functions as a simple decoy receptor in these Xenopus experiments.

A severe phenotype was found in three different mouse models, in which Fgfrl1 expression had been abolished by targeted gene disruption. The first knock-out mouse was described by Baertschi et al. [24]. These authors replaced the first two exons (the noncoding exon 0 and the first coding exon 1) by a neo cassette and studied the outcome in homozygous and heterozygous offspring. Heterozygous mice appeared to be normal as they did not display any special phenotype until 2 years after birth. Homozygous Fgfrl1 $1^{-} /^{-}$animals developed to term but died immediately after birth due to respiratory distress. The respiratory problems could be explained by a severe reduction of the diaphragm muscle, which was not strong enough to inflate the lungs after birth. The thickness of the diaphragm muscle reached barely $60 \%$ of that of the control animals, and certain areas of the diaphragm remained amuscular. Other skeletal muscles such as the muscles of the leg did not appear to be affected [24]. In addition to the diaphragm phenotype, homozygous knock-out mice showed subtle alterations in their skeleton, especially in their head [22]. They had a dome-shaped skull with high front that was reminiscent of certain mouse models for human craniosynostosis syndromes.

The most striking phenotype of the Fgfrl1 knock-out animals, however, was the virtual absence of the metanephric kidneys [47]. During early embryonic development, Fgfrl1 is normally expressed in the metanephric mesenchyme and in the nascent renal vesicles. At stage E10.5, the ureteric bud invades the metanephric mesenchyme; subsequently, it branches several times and induces the adjacent mesenchymal cells to undergo a mesenchymal-to-epithelial transition [48]. In the mutant animals, the ureteric bud showed severely reduced branching and the nephrogenic mesenchyme did not undergo the mesenchymal-to-epithelial transition. The failure of the induction of nephrons could be explained by a lack of expression of the nephrogenic markers Wnt4, Fgf8, Pax8, and Lim1 in the mutant kidney rudiments [47]. Thus, Fgfrll appears to be essential for the induction of the metanephric mesenchyme. It is of interest to note that other organs where branching morphogenesis plays a fundamental role, such as the lungs, did not appear to be affected in the mutant animals.

It is conceivable that part of the bone phenotype described above is secondary to the missing kidneys. Bilateral renal agenesis is known to cause oligohydramnios, a deficiency of amniotic fluid sometimes also referred 
to as the Potter syndrome or the Potter sequence [49]. Fetuses with Potter syndrome show many signs of intrauterine compression, including facial dysmorphia with large, low-set ears, redundant skin, limb-positioning defects, arthrogryposis, and lung hypoplasia. Skull ossification defects with large fontanels and sutures are a frequent component of the disease. It is likely that the missing kidneys will also affect the renin-angiotensin system (RAS) of the growing fetus since renin is usually produced in the afferent arterioles of the kidneys, even during fetal life [50]. When renin is absent from the circulation, angiotensinogen cannot be converted to angiotensin, and the fetus will experience life-threatening hypotension. Skull ossification defects are a common consequence of the low blood pressure since nascent bones require a high oxygen tension for normal growth [50]. It is therefore likely that at least part of the skeletal abnormalities of the Fgfrl1-deficient embryos can be explained by the missing kidneys.

A second mouse model was developed by Anderson et al. [51] and published as a patent application (US patent 20070292438). Although these authors did not provide any detailed description of the final targeting vector or any detailed analysis of the resulting phenotype, they clearly stated that the homozygous Fgfrl1 ${ }^{-} /^{-}$mice died around birth. Of note, their mice exhibited a fully penetrant kidney phenotype with bilateral kidney agenesis as described by Gerber et al. [47]. At embryonic stage E10.5 the ureteric bud of the Fgfrll-deficient mice invaded the metanephric mesenchyme, but then failed to branch to form the characteristic T-shaped structure. Expression of Pax2 and Wnt $11 \mathrm{~b}$ was severely reduced in the metanephric mesenchyme. The authors proposed that Fgfrl1 is one of the key regulators of early kidney development [51]. Since the phenotypes of the mice created by Baertschi et al. [24] and those created by Anderson et al. [51] are highly similar, bilateral kidney agenesis must be considered a general phenomenon of Fgfrl1 knock-out mice.

A third knock-out mouse has been created by Catela et al. [52]. These authors did not remove the promoter region but deleted the coding exons 2-6 of the Fgfrl1 gene. Similar to the mice of Baertschi et al. [24] and Anderson et al. [51], their heterozygous Fgfrl $1^{+} /^{-}$mice did not reveal any abnormalities. The homozygous $\mathrm{Fgfrl1}^{-} /^{-}$mice showed the severe diaphragm defects described above and died perinatally or even prenatally [52]. In addition, the authors observed hypoplasia of most skeletal elements, including shortened axial and appendicular bones and malformed vertebrae. Furthermore, the embryos had congenital heart defects such as thickened ventricular valves and septation anomalies. They suffered from a transient fetal anemia that was claimed to be the cause for the relatively high incidence of prenatal lethality. Finally, the authors suggested that their knock-out mice might provide a novel animal model to study the human Wolf-Hirschhorn syndrome (see also below). However, Catela et al. [52] did not mention any data about the missing kidneys. It is therefore not clear at the moment whether some of the observed alterations are actually secondary defects caused by the potentially lacking kidneys.

\section{FGFRL1 in human diseases}

The first human mutation in FGFRL1 was identified by Rieckmann et al. [22] in a craniosynostosis patient. Screening of the genomic DNA from 55 patients with various congenital skeletal malformations led to the identification of one patient with a frameshift mutation in the intracellular domain of FGFRL1. This female patient presented with craniosynostosis, radio-ulnar synostosis, and genital abnormalities and had previously been diagnosed with Antley-Bixler syndrome. She was heterozygous for an insertion of four nucleotides close to the $\mathrm{C}$ terminus of FGFRL1. The insertion caused an elongation of the polypeptide chain from 504 amino acid residues to 551 residues. In addition, this patient harbored two mutations in P450 oxidoreductase, an enzyme involved in the synthesis of several steroid hormones. Cell culture experiments showed that the mutant FGFRL1 protein stayed mainly at the plasma membrane where it could interact with FGF ligands, while the wild-type protein was preferentially located in vesicular structures and in the Golgi complex. The authors speculated that the mutation might lead to an overactivity of the FGFRL1 receptor and contribute in this way to the skeletal malformations of the patient [22]. In contrast to the three mouse models described above that show FGFRL1 loss-of-function, this patient would therefore represent an example with FGFRL1 gain-of-function. That FGFRL1 is indeed expressed in the growth plate of long bones had previously been demonstrated by Lazarus et al. [53]. These authors used microdissection followed by qPCR and showed that FGFRL1 was expressed not only in the perichondrium, but also in the growth plate of newborn rats, preferentially in the resting zone.

There are a few reports in the literature that implicate the FGFRL1 gene with the etiology of Wolf-Hirschhorn syndrome. The primary cause of Wolf-Hirschhorn syndrome is a hemizygous deletion of variable size in the short arm of chromosome 4 at $4 \mathrm{p} 16$ [54]. The major characteristics of the disease are growth delay, craniofacial dysgenesis, developmental delay, and epilepsy, but the phenotype is highly variable. Mapping of the chromosomal deletions from different patients identified two critical regions termed WHSCR-1 and WHSCR-2. Both regions comprise part of the WHSC1 gene, which was therefore 
made responsible for the craniofacial appearance of patients with Wolf-Hirschhorn syndrome. Recently, Engbers et al. [55] reported on a female patient with characteristics of Wolf-Hirschhorn syndrome. This patient had a terminal 4 p16.3 deletion that included the FGFRL1 gene, but did not include the WHSC1 gene. The authors therefore suggested that FGFRL1 might represent a plausible candidate for the facial abnormalities of some Wolf-Hirschhorn syndrome patients.

Catela et al. [52] resumed this idea and proposed that their Fgfrl1 knock-out mouse might provide a novel animal model for the dissection of the complex etiology of Wolf-Hirschhorn syndrome. Their mice showed abnormal craniofacial development, axial and appendicular skeletal anomalies, and congenital heart defects similar to Wolf-Hirschhorn syndrome patients. However, there are several pieces of evidence that would rather argue against this animal model. In human patients, hemizygosity of one or several genes in 4 p16.3 is the generally accepted cause for the craniofacial phenotype of Wolf-Hirschhorn syndrome [54], but none of the three Fgfrl1 knock-out mouse models showed any abnormal phenotype if only one allele of the Fgfrl1 gene was deleted (heterozygous mice) [24, 51, 52]. On the other hand, two of the Fgfrl1 knock-out mouse models displayed bilateral kidney agenesis as the major defect $[47,51]$, but kidney problems have not been associated with Wolf-Hirschhorn syndrome [54]. It should also be taken into consideration that the FGFRL1 locus is situated on 4p16.3 in close proximity to the FGFR3 locus. It is therefore possible that the deletion of a locus control region or of a remote enhancer element will influence the expression of FGFR3 even when this gene is not directly affected by the deletion.

Deletions in the chromosomal region 4 p16 are also found in some patients suffering from congenital diaphragmatic hernia [56], and there are several reports about Wolf-Hirschhorn syndrome patients with congenital diaphragmatic hernia. The smallest deletion from the end of the short arm of chromosome 4 associated with a diaphragmatic defect was determined as $2.4 \mathrm{Mb}$ [57]. This deletion encompasses more than 40 different genes including FGFRL1. Since Fgfrl1-deficient mice have a hypoplastic diaphragm, the FGFRL1 gene was searched for mutations in 54 patients with congenital diaphragmatic hernia. Six established polymorphisms were found, but no novel gene mutations [58]. The database of single nucleotide polymorphisms, dnSNP (http://www.ncbi.nlm.nih. gov/snp), lists seven nonsynonymous SNPs that lead to changes in the amino acid sequence (Table 1). Only three of these SNPs occur with significant frequency in the Caucasian population, namely P362Q, R424L, and P464L, and all these SNPs are located within the last exon of the FGFRL1 gene. One congenital diaphragmatic hernia patient was found to be hemizygous for the minor allele of two nonsynonymous SNPs (P362Q, P464L) [58]. It is therefore conceivable that these polymorphisms predispose the patient to diaphragmatic hernia. In this context, it is interesting to note that the P362Q polymorphism occurs at a site where the extracellular domain of FGFRL1 can be cleaved and shed from the membrane. In fact, cell culture experiments have demonstrated that the 362-Gln variant is cleaved considerably better than the 362-P variant [20]. It is therefore plausible that the 362-Gln variant and eventually also the 464-L variant affect the function of FGFRL1 in patients who are hemizygous for this region.

Finally, alterations in FGFRL1 expression have been observed in human tumors. A screen of 241 different human tumors identified significant alterations in the relative expression in ovarian tumors [59]. Five ovarian tumors were further analyzed by qPCR. While three tumors showed a significant decrease in FGFRL1 expression, one ovarian tumor exhibited a 25 -fold increase in the relative expression, suggesting major aberrations of FGFRL1 expression in ovarian cancer.

\section{Concluding remarks}

A new level of complexity was added to the FGF signaling system when the klotho gene family was discovered [60, 61]. The klotho proteins convert the canonical FGFR1 into a specific receptor for FGF23, FGF21, or FGF19, respectively [61]. FGFRL1 now appears to add a further level of complexity to the FGF signaling system. Although the exact function of FGFRL1 is far from being understood, it does represent a true player in the FGF signaling system since it is able to interact with FGF ligands and with heparin and since it evokes a cellular effect that ultimately leads to reduced cell growth and accelerated cell differentiation. While some of the properties of FGFRL1 are relatively easy to explain, others appear rather difficult to reconcile. For example, FGFRL1 binds to FGF2 as do all the other FGFRs, but it does not bind to FGF1, which is also recognized by all of the other FGFRs. The strongest expression of Fgfrl1 is observed in cartilage but mice with a targeted deletion of the Fgfrl1 gene do not show any severe cartilage phenotype. These mice rather exhibit a severe kidney phenotype with bilateral kidney agenesis, although Fgfrl1 is not expressed at particularly high levels in the developing kidneys. The expression levels of the kidneys certainly do not exceed those of the developing lungs, but the lungs appear to be normal in the mutant mice.

It is possible that the FGFRL1 gene is one of the first genes of the FGF/FGFR system that developed during 
Table 1 Coding nonsynonymous SNPs of the human FGFRL1 gene

\begin{tabular}{lllll}
\hline Position & Allele change & Residue change & Allele frequency & Population \\
\hline 1016006 & GCG $\rightarrow$ GTG & V32A & $0.980(n=50)$ & Black african \\
1016188 & GAT $\rightarrow$ AAT & D93N & $0.500(n=2)$ & Caucasian \\
1017749 & CGC $\rightarrow$ CTC & R193L & Not given & Not given \\
1018705 & CCA $\rightarrow$ CAA & P362Q & $0.725(n=218)$ & Caucasian \\
1018891 & CGC $\rightarrow$ CTC & R424L & $0.970(n=216)$ & Caucasian \\
1019010 & CCA $\rightarrow$ CGA & P464A & Not given $(n=8)$ & Not given \\
1019011 & CCA $\rightarrow$ CTA & P464L & $0.930(n=216)$ & Caucasian \\
\hline
\end{tabular}

evolution for it is already found in the first metazoans such as the sea anemone [18]. This observation strongly suggests that it might have evolved together with the other members of the FGFR family. One should also consider the possibility that FGFRL1 was even the first FGFR, which subsequently gave rise to the evolution of the classical FGFRs by acquiring a tyrosine kinase domain from an unrelated receptor. The relative age of the FGFRL1 gene may partly explain the fact that this receptor adopted several seemingly different functions that are difficult to reconcile to date. Besides its effects on cell growth, it also promotes cell adhesion and cell-cell fusion, two properties that so far have not been observed with any of the canonical FGFRs.

Recently, FGFRL1 caught the attention of researchers from different fields of biology because it repeatedly emerged as one of the best candidates from several genome-wide screens. For example, FGFRL1 turned up in an approach to identify novel human genes that show genomic imprinting [62]. Imprinted genes are essential for the early development of an embryo and dysregulation of imprinting can lead to severe human diseases such as Prader-Willi syndrome [63]. The authors of that study utilized a novel algorithm to predict that FGFRL1 is expressed only from the maternal allele, yet experimental verification of this prediction is still missing [62]. A microarray-based search for genes involved in self-renewal of embryonic stem cells revealed that FGF signaling is one of the master regulators of sustained self-renewal [64]. This study identified FGFRL1 as one of the top candidates that were up-regulated in response to RNAi-mediated silencing of the core transcription factors OCT4 and SOX2. Finally, a study aiming at the identification of specific hypoxiaregulated miRNAs detected mir-210 as the most prominent miRNA induced under hypoxic conditions [65]. Interestingly, one of the target genes of mir-210 that harbors multiple mir-210 binding sites in its $3^{\prime}$ UTR turned out to be FGFRL1. These authors further demonstrated that overexpression of mir-210 reduced the growth of tumors when cells from a head-and-neck tumor were injected into immunodeficient mice. Interestingly, this inhibition could be overcome by stable co-expression of FGFRL1 in the injected cells [65]. Although the relevance of all these observations is not yet fully clear, they nevertheless demonstrate that the function of FGFRL1 goes well beyond that of a mere decoy receptor for FGF ligands.

Acknowledgments Research in the laboratory of the author was supported by grants from the Swiss National Science Foundation (3100A-127046) and from the Swiss Foundation for Research on Muscular Diseases.

\section{References}

1. Beenken A, Mohammadi M (2009) The FGF family: biology, pathophysiology and therapy. Nat Rev Drug Discov 8:235-253

2. Itoh N, Ornitz DM (2004) Evolution of the Fgf and Fgfr gene families. Trends Genet 20:563-569

3. Eswarakumar VP, Lax I, Schlessinger J (2005) Cellular signaling by fibroblast growth factor receptors. Cytokine Growth Factor Rev 16:139-149

4. Mohammadi M, Olsen SK, Ibrahimi OA (2005) Structural basis for fibroblast growth factor receptor activation. Cytokine Growth Factor Rev 16:107-137

5. Wilkie AO (2005) Bad bones, absent smell, selfish testes: the pleiotropic consequences of human FGF receptor mutations. Cytokine Growth Factor Rev 16:187-203

6. Knowles MA (2007) Role of FGFR3 in urothelial cell carcinoma: biomarker and potential therapeutic target. World J Urol 25:581-593

7. Jackson CC, Medeiros LJ, Miranda RN (2010) 8p11 myeloproliferative syndrome: a review. Hum Pathol 41:461-476

8. Wiedemann M, Trueb B (2000) Characterization of a novel protein (FGFRL1) from human cartilage related to FGF receptors. Genomics 69:275-279

9. Kim I, Moon S-O, Yu K-H, Kim U-H, Koh GY (2001) A novel fibroblast growth factor receptor-5 preferentially expressed in the pancreas. Biochim Biophys Acta 1518:152-156

10. Sleeman M, Fraser J, McDonald M, Yuan S, White D, Grandison P, Kumble K, Watson JD, Murison JG (2001) Identification of a new fibroblast growth factor receptor, FGFR5. Gene 271:171-182

11. Trueb B, Zhuang L, Taeschler S, Wiedemann M (2003) Characterization of FGFRL1, a novel FGF receptor preferentially expressed in skeletal tissues. J Biol Chem 278:33857-33865

12. Trueb B, Taeschler S (2006) Expression of FGFRL1, a novel fibroblast growth factor receptor, during embryonic development. Int J Mol Med 17:617-620

13. Wiedemann M, Trueb B (2001) The mouse Fgfrl1 gene coding for a novel FGF receptor-like protein. Biochim Biophys Acta 1520:247-250 
14. Hayashi S, Itoh M, Taira S, Agata K, Taira M (2004) Expression patterns of Xenopus FGF receptor-like 1/nou-darake in early Xenopus development resemble those of planarian nou-darake and Xenopus FGF8. Dev Dyn 230:700-707

15. Beyeler M, Trueb B (2006) Fgfrl1, a fibroblast growth factor receptor-like gene, is found in the cephalochordate Branchiostoma floridae but not in the urochordate Ciona intestinalis. Comp Biochem Physiol Part B 145:43-49

16. Trueb B, Neuhauss SCF, Baertschi S, Rieckmann T, Schild C, Taeschler S (2005) Fish possess multiple copies of fgfrl1, the gene for a novel FGF receptor. Biochim Biophys Acta 1727:65-74

17. Zhuang L, Karotki AV, Bruecker P, Trueb B (2009) Comparison of the receptor FGFRL1 from sea urchins and humans illustrates evolution of a zinc binding motif in the intracellular domain. BMC Biochemistry 10:33

18. Bertrand S, Somorjai I, Garcia-Fernandez J, Lamonerie T, Escriva H (2009) FGFRL1 is a neglected putative actor of the FGF signalling pathway present in all major metazoan phyla. BMC Evol Biol 9:226

19. Putnam NH, Srivastava M, Hellsten U, Dirks B, Chapman J, Salamov A, Terry A, Shapiro H, Lindquist E, Kapitonov VV, Jurka J, Genikhovich G, Grigoriev IV, Lucas SM, Steele RE, Finnerty JR, Technau U, Martindale MQ, Rokhsar DS (2007) Sea anemone genome reveals ancestral eumetazoan gene repertoire and genomic organization. Science 317:86-94

20. Steinberg F, Zhuang L, Beyeler M, Kalin RE, Mullis PE, Brandli AW, Trueb B (2010) The FGFRL1 receptor is shed from cell membranes, binds FGFs and antagonizes FGF signaling in Xenopus embryos. J Biol Chem 285:2193-2202

21. Rieckmann T, Kotevic I, Trueb B (2008) The cell surface receptor FGFRL1 forms constitutive dimers that promote cell adhesion. Exp Cell Res 314:1071-1081

22. Rieckmann T, Zhuang L, Flück CE, Trueb B (2009) Characterization of the first FGFRL1 mutation identified in a craniosynostosis patient. Biochim Biophys Acta 1792:112-121

23. Zhuang L, Falquet L, Trueb B (2010) Genome-wide comparison of FGFRL1 with structurally related surface receptors. Exp Ther Med 1:161-168

24. Baertschi S, Zhuang L, Trueb B (2007) Mice with a targeted disruption of the Fgfrl1 gene die at birth due to alterations in the diaphragm. FEBS J 274:6241-6253

25. Plotnikov AN, Schlessinger J, Hubbard SR, Mohammadi M (1999) Structural basis for FGF receptor dimerization and activation. Cell 98:641-650

26. Plotnikov AN, Hubbard SR, Schlessinger J, Mohammadi M (2000) Crystal structures of two FGF-FGFR complexes reveal the determinants of ligand-receptor specificity. Cell 101:413-424

27. Ibrahimi OA, Zhang F, Eliseenkova AV, Itoh N, Linhardt RJ, Mohammadi M (2004) Biochemical analysis of pathogenic ligand-dependent FGFR2 mutations suggests distinct pathophysiological mechanisms for craniofacial and limb abnormalities. Hum Mol Genet 13:2313-2324

28. Mohammadi M, Olsen SK, Goetz R (2005) A protein canyon in the FGF-FGF receptor dimer selects from an à la carte menu of heparan sulfate motifs. Curr Opin Struct Biol 15:506-516

29. Kan M, Wang F, Xu J, Crabb JW, Hou J, McKeehan WL (1993) An essential heparin-binding domain in the fibroblast growth factor receptor kinase. Science 259:1918-1921

30. Powell AK, Fernig DG, Turnbull JE (2002) Fibroblast growth factor receptors 1 and 2 interact differently with heparin/heparan sulfate. Implications for dynamic assembly of a ternary signaling complex. J Biol Chem 277:28554-28563

31. Loo BM, Kreuger J, Jalkanen M, Lindahl U, Salmivirta M (2001) Binding of heparin/heparan sulfate to fibroblast growth factor receptor 4. J Biol Chem 276:16868-16876
32. Mantovani A, Locati M, Vecchi A, Sozzani S, Allavena P (2001) Decoy receptors: a strategy to regulate inflammatory cytokines and chemokines. Trends Immunol 22:328-336

33. Davis RS (2007) Fc receptor-like molecules. Annu Rev Immunol 25:525-560

34. Long EO (2008) Negative signaling by inhibitory receptors: the NK cell paradigm. Immunol Rev 224:70-84

35. Crocker PR, Redelinghuys P (2008) Siglecs as positive and negative regulators of the immune system. Biochem Soc Trans 36:1467-1471

36. Daeron M, Jaeger S, Du Pasquier L, Vivier E (2008) Immunoreceptor tyrosine-based inhibition motifs: a quest in the past and future. Immunol Rev 224:11-43

37. Pinheiro da Silva F, Aloulou M, Benhamou M, Monteiro RC (2008) Inhibitory ITAMs: a matter of life and death. Trends Immunol 29:366-373

38. Steinberg F, Gerber S, Rieckmann T, Trueb B (2010) Rapid fusion and syncytium formation of heterologous cells upon expression of the FGFRL1 receptor. J Biol Chem 285. doi: 10.1074/jbc.M110.140517

39. Chen EH, Grote E, Mohler W, Vignery A (2007) Cell-cell fusion. FEBS Lett 581:2181-2193

40. Oren-Suissa M, Podbilewicz B (2010) Evolution of programmed cell fusion: common mechanisms and distinct functions. Dev Dyn 239:1515-1528

41. Cebria F, Kobayashi C, Umesono Y, Nakazawa M, Mineta K, Ikeo K, Gojobori T, Itoh M, Taira M, Sanchez Alvarado A, Agata K (2002) FGFR-related gene nou-darake restricts brain tissues to the head region of planarians. Nature 419:620-624

42. Ogawa K, Kobayashi C, Hayashi T, Orii H, Watanabe K, Agata K (2002) Planarian fibroblast growth factor receptor homologs expressed in stem cells and cephalic ganglions. Dev Growth Differ 44:191-204

43. Hall C, Flores MV, Murison G, Crosier K, Crosier P (2006) An essential role for zebrafish Fgfrl1 during gill cartilage development. Mech Dev 123:925-940

44. Hogan BM, Hunter MP, Oates AC, Crowhurst MO, Hall NE, Heath JK, Prince VE, Lieschke GJ (2004) Zebrafish gcm2 is required for gill filament budding from pharyngeal ectoderm. Dev Biol 276:508-522

45. Hanaoka R, Ohmori Y, Uyemura K, Hosoya T, Hotta Y, Shirao T, Okamoto H (2004) Zebrafish gcmb is required for pharyngeal cartilage formation. Mech Dev 121:1235-1247

46. Amaya E, Stein PA, Musci TJ, Kirschner MW (1993) FGF signalling in the early specification of mesoderm in Xenopus. Development 118:477-487

47. Gerber S, Steinberg F, Beyeler M, Villiger PM, Trueb B (2009) The murine Fgfrl1 receptor is essential for the development of the metanephric kidney. Dev Biol 335:106-119

48. Saxen L, Sariola H (1987) Early organogenesis of the kidney. Pediatr Nephrol 1:385-392

49. Potter EL (1962) Pathology of the fetus and infant, 2nd edn. Year Book Medical, Chicago

50. Gubler MC, Antignac C (2010) Renin-angiotensin system in kidney development: renal tubular dysgenesis. Kidney Int 77:400-406

51. Anderson SJ, Brennan J, de Sauvage FJ, Ding Z, Edwards J, Fikes NA, Huang W, Ouyang W, Rangel C, Sangha M, Shi Z-Z, Sparks MJ, Trackey J, Vetter M, Wang C-Y, Woodings J (2007) Novel gene disruptions, compositions and methods relating thereto. US Patent 20070292438

52. Catela C, Bilbao-Cortes D, Slonimsky E, Kratsios P, Rosenthal N, Te Welscher P (2009) Multiple congenital malformations of WolfHirschhorn syndrome are recapitulated in Fgfrl1 null mice. Dis Model Mech 2:283-294 
53. Lazarus JE, Hegde A, Andrade AC, Nilsson O, Baron J (2007) Fibroblast growth factor expression in the postnatal growth plate. Bone 40:577-586

54. Battaglia A, Carey JC, Wright TJ (2001) Wolf-Hirschhorn (4p-) syndrome. Adv Pediatr 48:75-113

55. Engbers H, van der Smagt JJ, van 't Slot R, Vermeesch JR, Hochstenbach R, Poot M (2009) Wolf-Hirschhorn syndrome facial dysmorphic features in a patient with a terminal $4 \mathrm{p} 16.3$ deletion telomeric to the WHSCR and WHSCR 2 regions. Eur $\mathbf{J}$ Hum Genet 17:129-132

56. Holder AM, Klaassens M, Tibboel D, de Klein A, Lee B, Scott DA (2007) Genetic factors in congenital diaphragmatic hernia. Am J Hum Genet 80:825-845

57. Casaccia G, Mobili L, Braguglia A, Santoro F, Bagolan P (2006) Distal $4 \mathrm{p}$ microdeletion in a case of Wolf-Hirschhorn syndrome with congenital diaphragmatic hernia. Birth Defects Res A Clin Mol Teratol 76:210-213

58. Lopez Jiminez N, Gerber S, Popovici V, Mirza S, Copren K, Ta L, Shaw GM, Trueb B, Slavotinek AM (2010) Examination of FGFRL1 as a candidate gene for diaphragmatic defects at chromosome 4p16.3 shows that Fgfrl1 null mice have reduced expression of Tpm3, sarcomere genes and Lrtm1 in the diaphragm. Hum Genet 127:325-336

59. Schild C, Trueb B (2005) Aberrant expression of FGFRL1, a novel FGF receptor, in ovarian tumors. Int $\mathrm{J}$ Mol Med 16:1169-1173
60. Urakawa I, Yamazaki Y, Shimada T, Iijima K, Hasegawa H, Okawa K, Fujita T, Fukumoto S, Yamashita T (2006) Klotho converts canonical FGF receptor into a specific receptor for FGF23. Nature 444:770-774

61. Kurosu H, Kuro-O M (2009) The Klotho gene family as a regulator of endocrine fibroblast growth factors. Mol Cell Endocrinol 299:72-78

62. Luedi PP, Dietrich FS, Weidman JR, Bosko JM, Jirtle RL, Hartemink AJ (2007) Computational and experimental identification of novel human imprinted genes. Genome Res 17:1723-1730

63. Falls JG, Pulford DJ, Wylie AA, Jirtle RL (1999) Genomic imprinting: implications for human disease. Am J Pathol 154:635-647

64. Greber B, Lehrach H, Adjaye J (2007) Silencing of core transcription factors in human EC cells highlights the importance of autocrine FGF signaling for self-renewal. BMC Dev Biol 7:46

65. Huang X, Ding L, Bennewith KL, Tong RT, Welford SM, Ang KK, Story M, Le QT, Giaccia AJ (2009) Hypoxia-inducible mir-210 regulates normoxic gene expression involved in tumor initiation. Mol Cell 35:856-867 